\title{
Yes-Associated Protein Expression Is Correlated to the Differentiation of Prostate Adenocarcinoma
}

\author{
Myung-Giun Noh · Sung Sun Kim \\ Eu Chang Hwang ${ }^{1}$ \\ Dong Deuk Kwon ${ }^{1}$ · Chan Choi \\ Departments of Pathology and ${ }^{1}$ Urology, \\ Chonnam National University Hwasun Hospital, \\ Chonnam National University Medical School, \\ Hwasun, Korea
}

\author{
Received: March 15, 2017 \\ Revised: April 17, 2017 \\ Accepted: May 4, 2017 \\ Corresponding Author \\ Chan Choi, MD, PhD \\ Department of Pathology, Chonnam National \\ University Hwasun Hospital, Chonnam National \\ University Medical School, 322 Seoyang-ro, \\ Hwasun 58128, Korea \\ Tel: +82-61-379-7071 \\ Fax: +82-61-379-7099 \\ E-mail: cchoi@chonnam.ac.kr
}

\begin{abstract}
Background: Yes-associated protein (YAP) in the Hippo signaling pathway is a growth control pathway that regulates cell proliferation and stem cell functions. Abnormal regulation of YAP was reported in human cancers including liver, lung, breast, skin, colon, and ovarian cancer. However, the function of YAP is not known in prostate adenocarcinoma. The purpose of this study was to investigate the role of YAP in tumorigenesis, differentiation, and prognosis of prostate adenocarcinoma. Methods: The nuclear and cytoplasmic expression of YAP was examined in 188 cases of prostate adenocarcinoma using immunohistochemistry. YAP expression levels were evaluated in the nucleus and cytoplasm of the prostate adenocarcinoma and the adjacent normal prostate tissue. The presence of immunopositive tumor cells was evaluated and interpreted in comparison with the patients' clinicopathologic data. Results: YAP expression levels were not significantly different between normal epithelial cells and prostate adenocarcinoma. However, YAP expression level was significantly higher in carcinomas with a high Gleason grades (8-10) than in carcinomas with a low Gleason grades $(6-7)(p<.01)$. There was no statistical correlation between YAP expression and stage, age, prostate-specific antigen level, and tumor volume. Biochemical recurrence $(B C R)-$ free survival was significantly lower in patients with high YAP expressing cancers $(p=.02)$. However high YAP expression was not an independent prognostic factor for BCR in the Cox proportional hazards model. Conclusions: The results suggested that YAP is not associated with prostate adenocarcinoma development, but it may be associated with the differentiation of the adenocarcinoma. YAP was not associated with BCR.
\end{abstract}

Key Words: Yes-associated protein (YAP); Prostate adenocarcinoma; Immunohistochemistry; Gleason score
Prostate cancer (PC) remains a leading cause of cancer-related death in North-American men ${ }^{1}$ and is becoming an increasingly common cancer in South Korea. ${ }^{2}$ Pathological stage and Gleason grade are important predictors of prognosis in patients with primary prostate adenocarcinoma who undergo a radical prostatectomy. The Gleason grading system is a well-established system for prostate adenocarcinoma that correlates with the differentiation of the prostate gland, pathological stage, disease progression, and recurrence. ${ }^{3,4}$ However, prostate adenocarcinoma is a remarkably heterogeneous disease. Distinguishing tumors associated with a poor outcome at the time of radical prostatectomy is problematic. The molecular mechanisms of prostate carcinogenesis remain poorly understood. ${ }^{5}$

The Hippo pathway, a vital growth regulator of cell proliferation and apoptosis, was first identified by mosaic screens in Drosophila melanogaster. ${ }^{6}$ Information about the Hippo pathway in Drosophila is likely applicable directly to mammalian systems, as it has been shown that mammalian homologues are capable of rescuing Drosophila mutants defective in the Hippo signaling pathway. ${ }^{7}$ Yes-associated protein (YAP) is a transcriptional coactivator of the Hippo pathway and is a highly conserved component of this pathway in mammalian systems. In humans, amplification of the chromosomal region containing the YAP gene (11q22) has been reported in several tumor types. ${ }^{8}$ Recent genetic mouse models and studies with cancer patient demonstrated the critical roles of Hippo-YAP signaling in cancer development. For examples, immunohistochemistry studies have shown that an elevated expression/nuclear localization of YAP or transcriptional coactivator with a PDZ-binding domain (TAZ) correlates with malignant features in lung cancer. ${ }^{9}$ In datasets of breast cancer patients, elevated expression of gene signatures for YAP/TAZ activity correlates with high histological grade, enrichment of stem cell signatures, metastasis proclivity, and poor outcome. ${ }^{10,11}$ High expression of YAP activity has been found to be prognostic for bad outcome in four datasets of colorectal cancer patients and correlated with cetuximab resistance. ${ }^{12}$ Immunohistochemistry 
studies on human hepatocellular carcinoma samples showed that elevated expression of YAP or TAZ correlates with poor tumor differentiation and is prognostic of bad outcome. ${ }^{13}$ By immunohistochemistry on human pancreatic tissue samples, YAP and TAZ were found to be almost absent from normal acini, but moderately expressed and nuclearly localized in PanINs and in a subset of primary pancreatic ductal adenocarcinoma, whereas strong nuclear staining of YAP was found in metastases derived from pancreatic adenocarcinoma. ${ }^{14}$

Although the net effect of deregulated YAP and TAZ activities in many tissues is similar, their activities appear to be controlled by different regulatory mechanisms in different tissues. ${ }^{15}$ Previous studies demonstrated the biological significance of the HippoYAP signaling pathway in prostate adenocarcinoma, but largescale studies have failed to identify YAP amplification and mutations in castration resistant PCs. ${ }^{16}$ Zhang et al. ${ }^{3}$ showed that the Hippo effector YAP regulates cell motility, invasion, and castration-resistant growth of prostate adenocarcinoma in rats. Hu et $a l{ }^{17}$ showed that YAP expression in PC is inversely correlated with increase in Gleason score. However, the clinical significance of YAP amplification in human prostate adenocarcinoma has largely remained unknown. This study investigates the role of YAP in the development, differentiation, and prognosis of prostate adenocarcinoma.

\section{MATERIALS AND METHODS}

\section{Patients and tumor samples}

Prostate acinar adenocarcinoma specimens were obtained from 188 patients who had undergone radical prostatectomy at Chonnam National University Hwasun Hospital from 2005 to 2012. The availability of adequate tissue material was the only inclusion criterion. Diagnostic criteria of prostate acinar adenocarcinoma were in agreement with the World Health Organization classification. Clinicopathologic data were collected from the medical records. All patients were advised to have prostatespecific antigen (PSA) follow-up every 3 months in the first year postoperation and at least biannually thereafter. Biochemical recurrence (BCR) was defined as two consecutive PSA measurements $\geq 0.2 \mathrm{ng} / \mathrm{mL}$ within an interval of more than 3 months. PSA progression-free survival time was defined as the time from radical prostatectomy to the first follow-up date showing PSA $\geq 0.2 \mathrm{ng} /$ $\mathrm{mL}$ or until the last follow-up. This study was approved by the Institutional Review Board of Chonnam National University Hwasun Hospital (CNUHH-2017-022).

\section{Immunohistochemistry for YAP}

One representative slide of the prostate adenocarcinoma was selected for immunohistochemical staining. Tumor samples obtained during surgical treatment were fixed in formalin and embedded in paraffin for histologic studies. The slides of the tumor surgical specimens were stained with hematoxylin and eosin and were reviewed and representative tissue blocks were selected. YAP (1A12) mouse monoclonal antibody (Cell Signaling Technology, Danvers, MA, USA) at a dilution of 1:200 was used for immunohistochemical analysis. Immunostaining was performed using the avidin-biotin complex method. Briefly, representative paraffin blocks were cut consecutively at $4-\mu \mathrm{m}$ thickness, and immunohistochemical staining was carried out using a BONDMAX Automated IHC/ISH Stainer (Leica Biosystems, Wetzlar, Germany).

Sections were deparaffinized in xylene and treated with $0.3 \%$ hydrogen peroxide in methanol for 20 minutes to block any endogenous peroxidase activity. Citrate buffer was used for antigen retrieval. Nonspecific binding was limited by using protein blocking buffer for 10 minutes. The sections were washed in phosphate-buffered saline and then incubated with the primary antibody for 20 minutes at room temperature. The samples were then incubated in secondary antibody (biotinylated) for 10 minutes, followed by incubation with streptavidin-horseradish peroxidase for 10 minutes, and exposed to diaminobenzidine, which was used as a chromogen. All labeled streptavidin-biotin-horseradish peroxidase system chemicals were obtained from Dako Cytomation Corp. (Carpinteria, CA, USA). Counterstaining was performed with Mayer's hematoxylin. Negative controls were treated similarly with the exception of incubation with the primary antibody (nonspecific staining control).

\section{Assessment of protein expression}

All immunostained slides were evaluated twice by two independent investigators blinded to the clinical details. Five views of the highest expression site were examined per slide, and 100 cells were observed per view at $400 \times$ magnification. In each slide, adjacent noncancerous basal cells of the prostate acinar tissue were available as internal positive controls and scattered lymphocyte were used as internal negative controls. YAP expression was graded according to the distribution, intensity, and percentage of positive cells as described previously. ${ }^{8}$ Both the tumor cells and noncancerous acinar cells were graded (Fig. 1). For the cytoplasmic distribution, weak cytoplasmic reactivity was considered as low expression regardless of the extent. Strong cytoplasmic reactivity with less than $50 \%$ positive cells was graded as low 


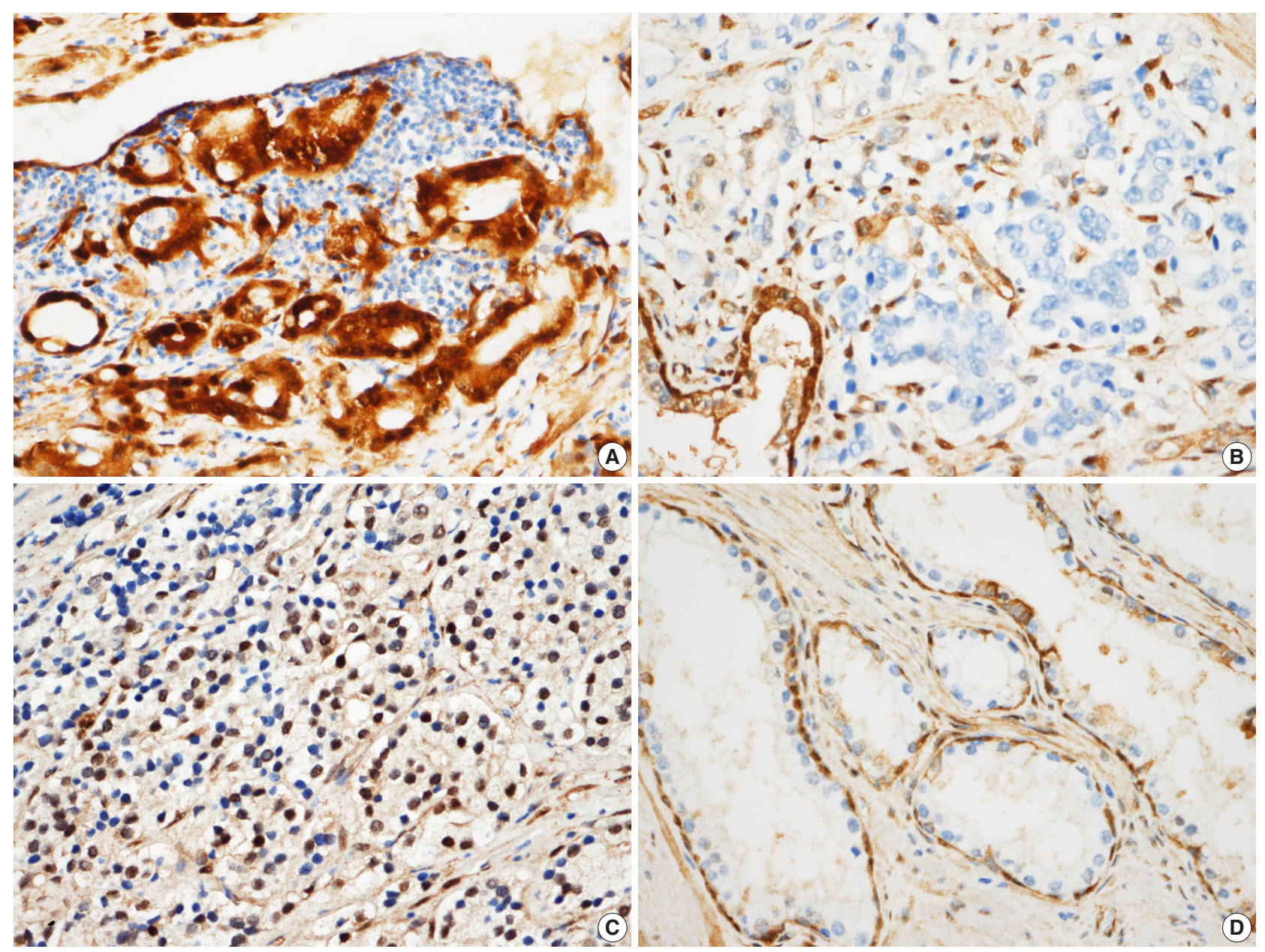

Fig. 1. Scoring according to the expression of Yes-associated protein (YAP) in prostate adenocarcinoma. (A) In prostate adenocarcinoma, both nuclei and cytoplasm are strongly positive for YAP, scored as high. (B) Negative expression is noted for YAP in prostate adenocarcinoma cells, which is scored as negative. (C) Nuclear expression in prostate adenocarcinoma cells, the nuclear stain over one tenth is scored as high. (D) YAP expression in normal prostate glands. Basal cells are strongly positive which were used as a positive control. Luminal cells did not show YAP expression. Stromal cells reveal mild expression of YAP.

expression; otherwise, it was graded as high expression if there were greater than $50 \%$ positive cells. For the nuclear distribution, samples with nuclear staining in less than $10 \%$ of cells were graded as low YAP expression, and samples with nuclear staining in more than $10 \%$ of cells were graded as high YAP high expression. YAP expression levels in prostate tissues were divided into high YAP expression and low/negative YAP expression. Two pathologists reviewed cases of inconclusive samples together and reached an agreement as to the YAP expression level.

\section{Gene Expression Omnibus data}

Microarray data of prostate adenocarcinoma deposited in the National Center for Biotechnology Information (NCBI) Gene Expression Omnibus (GEO) were analyzed.

\section{Statistical analysis}

Statistical analyses were performed with the statistical computing environment $\mathrm{R}$ (ver. 3.3.2, $\mathrm{R}$ Core Team) and the software program IBM SPSS (ver. 21.0 for Windows, IBM Corp., Armonk, NY, USA). Correlation with tumor and adjacent nontumor tissue in prostate samples was assessed by the Spearman's rank correlation coefficient test. Association between the Gleason grade and clinical factors on radical prostatectomy specimens with YAP expression was calculated using chi-square test. Statistically significant trends associated with an increasing Gleason grade and YAP expression were analyzed by Cochran Armitage trend test. Multivariate logistic regression analysis was used to develop a trend score for tumor differentiation based on patient characteristics (age, initial PSA tumor volume, YAP expression, and pathologic stage). Kaplan-Meier survival curves were used to 
assess the prognostic significance of YAP in predicting BCR. A multivariate analysis was performed using the Cox regression model to study the effects of different variables on BCR. All pvalues were based on two-tailed statistical analyses and $\mathrm{p}<.05$ was considered to be statistically significant. In GEO data, the Mann-Whitney and paired Wilcoxon tests were utilized when parameters were not normally distributed, while independentsamples and paired-sample $t$ tests were used when parameters were normally distributed. The Kolmogorov-Smirnov test was employed to demonstrate deviation from the normal distribution.

\section{RESULTS}

YAP expression in 188 cases of prostate adenocarcinoma and their paired adjacent nontumor tissues was investigated by immunohistochemistry. In the cytoplasm of normal prostate luminal cells, 45 cases (23.9\%) were completely negative, 125 cases (66.5\%) showed low expression in cytoplasm, and 18 cases (9.6\%) showed high expression. In the nucleus, 140 cases (74.4\%) were negative, 33 cases $(17.6 \%)$ showed low expression, and 15 cases (8.0\%) showed high expression (Table 1, Fig. 2A-D). In the nucleus of

Table 1. YAP expression in prostate adenocarcinoma and normal prostate tissue adjacent to the adenocarcinoma

\begin{tabular}{lcc}
\hline \multicolumn{1}{c}{ Type } & $\begin{array}{c}\text { Prostate } \\
\text { adenocarcinoma }\end{array}$ & $\begin{array}{c}\text { Normal prostate } \\
\text { glands adjacent to } \\
\text { adenocarcinoma }\end{array}$ \\
\hline $\begin{array}{l}\text { Complete absence of reactivity } \\
\text { Nucleus }\end{array}$ & $16 / 188(8.5)$ & $45 / 188(23.9)$ \\
Absent & $44 / 188(23.4)$ & $95 / 188(50.5)$ \\
Low & $84 / 188(44.7)$ & $33 / 188(17.6)$ \\
High & $10 / 188(5.3)$ & $7 / 188(3.7)$ \\
$\begin{array}{l}\text { Cytoplasm } \\
\text { Absent }\end{array}$ & \\
Low & $9 / 188(4.8)$ & $0 / 188(0)$ \\
High & $124 / 188(66.0)$ & $125 / 188(66.5)$ \\
Nucleus and cytoplasm & $5 / 188(2.7)$ & $10 / 188(5.3)$ \\
High & & \\
\hline
\end{tabular}

Values are presented as number (\%). YAP, Yes-associated protein.

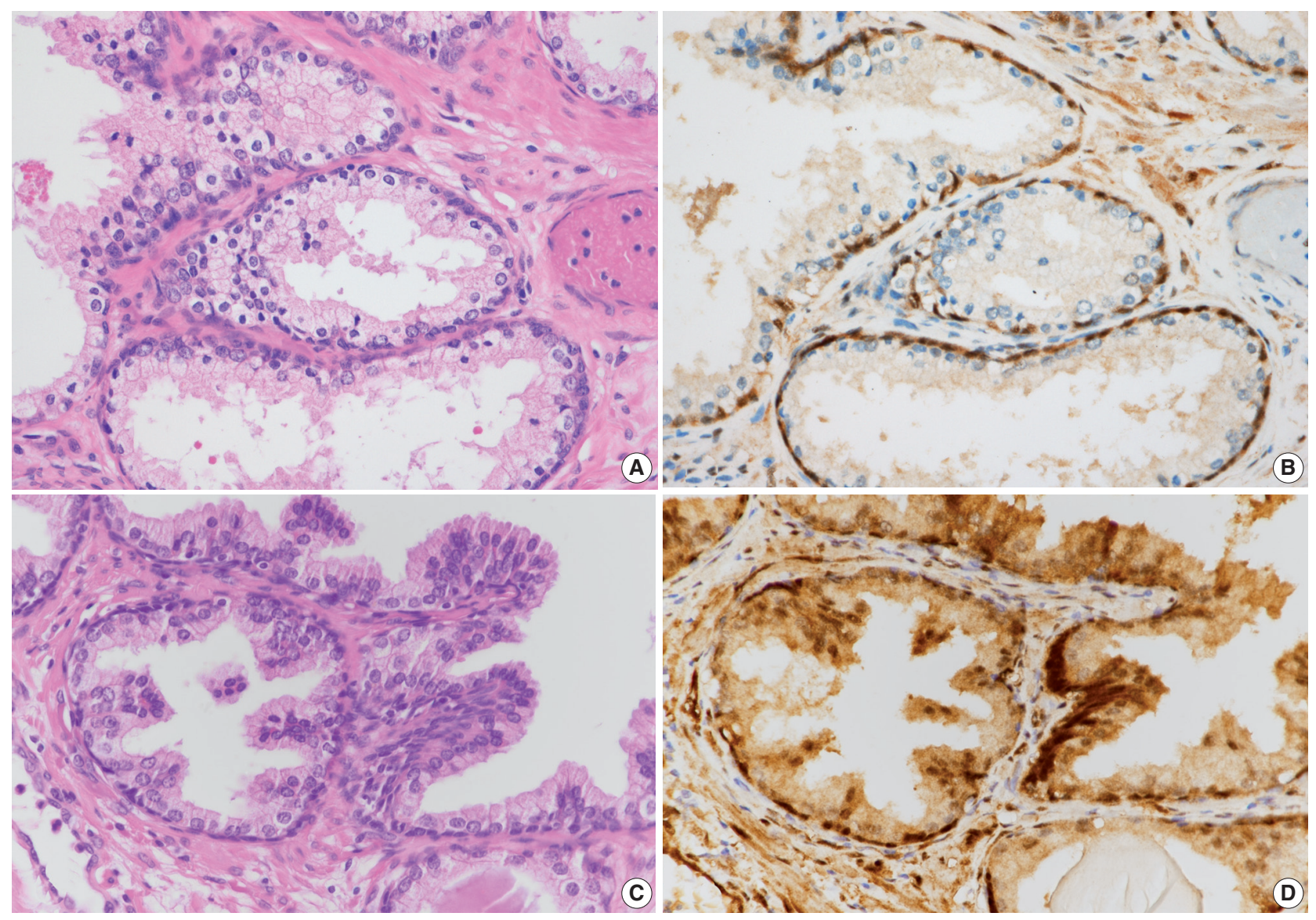

Fig. 2. Expression of Yes-associated protein (YAP) in prostate adenocarcinoma and normal prostate glands. (A-D) Hematoxylin and eosin (H\&E)-stained section of benign glands and immunohistochemical stain of YAP in normal prostate glands. Luminal cells of normal prostate gland show no expression to mild expression of YAP in the cytoplasm. All of the basal cells and stroma show positive YAP.

(Continued to the next page) 

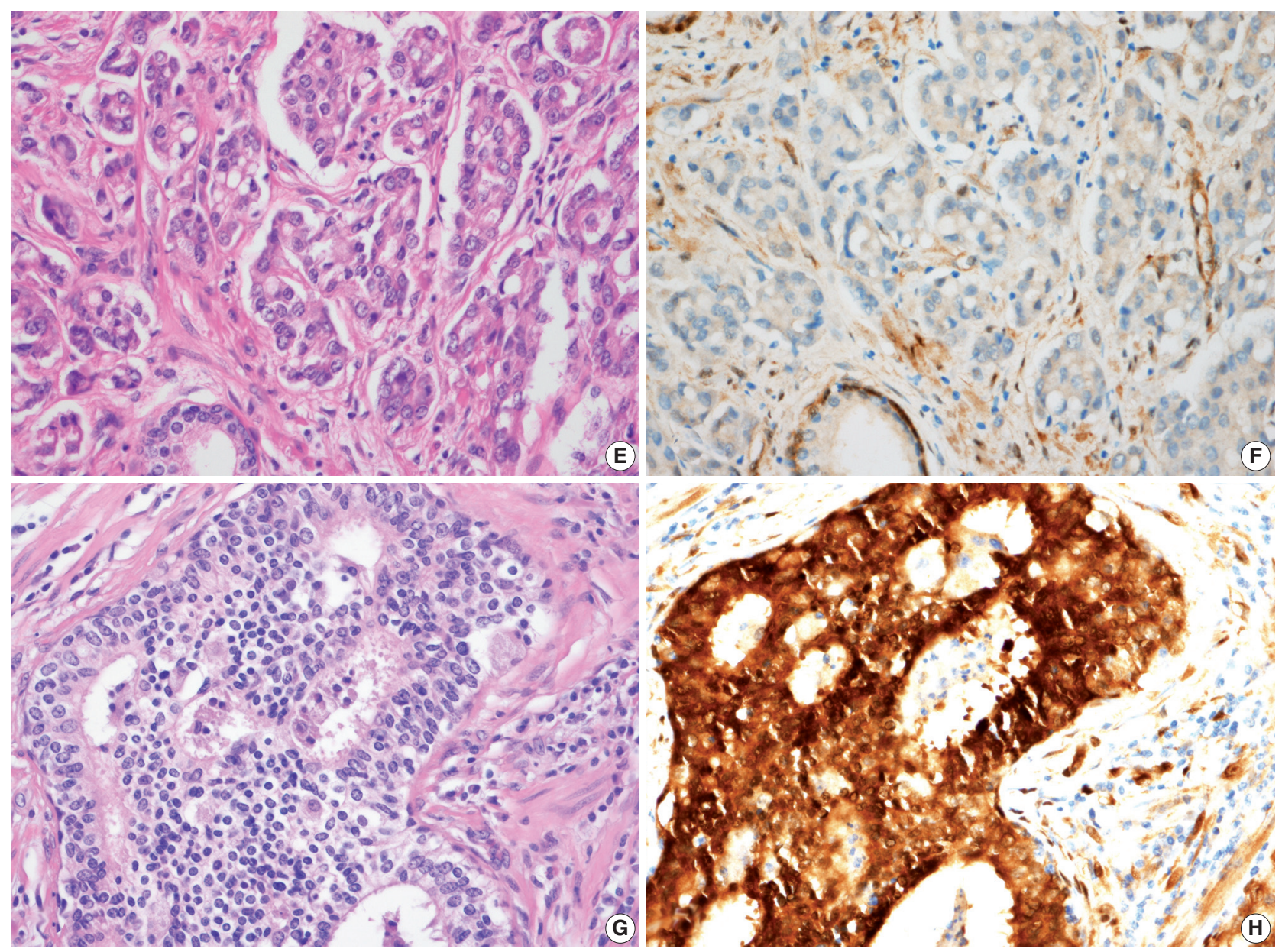

Fig. 2. (Continued from the previous page) (E) H\&E-stained section of well-to-moderately differentiated prostate adenocarcinoma (Gleason score, $4+3=7$ ). There are poorly formed glands and a few well-formed glands. (F) Expression of YAP in well-to-moderately differentiated prostate adenocarcinoma (Gleason score, $4+3=7$ ). Tumor cells show either no or mild expression in the cytoplasm. (G) H\&E-stained section of poorly differentiated prostate adenocarcinoma (Gleason score, $5+5=10)$. $(H)$ Tumor cells display strong positivity in both nuclei and cytoplasm.

prostate adenocarcinoma, 60 cases $(31.9 \%)$ were negative, 84 cases $(44.7 \%)$ showed low expression and 44 cases $(23.4 \%)$ were high expression. In the cytoplasm, 25 cases (13.3\%) were negative, 124 cases $(66.0 \%)$ showed low expression, and 39 cases $(20.7 \%)$ showed high expression (Table 1, Fig. 2E-H). Basal cells and stroma were positive for YAP (Fig. 2). ${ }^{17}$

According to the YAP immunohistochemical scoring system used in this study, 49 cases of prostate adenocarcinoma tissues (26.1\%) highly expressed YAP in both the nuclei and cytoplasm, whereas 139 cases of adenocarcinoma tissues $(73.9 \%)$ were negative/ low positive for YAP expression (Table 2). In contrast, 163 cases $(86.7 \%)$ revealed low/negative expression of YAP and only 25 cases of these normal cells $(13.3 \%)$ were highly positive for YAP expression (Table 2). The expression of YAP was not significantly different between tumor and adjacent normal tissues in the prostate adenocarcinoma samples ( $\mathrm{p}>$.05) (Table 2).

YAP mRNA expression level in prostate adenocarcinoma available from GEO profiles was evaluated. ${ }^{18-24}$ Only one study found YAP expression to be higher in prostate adenocarcinoma than in normal tissue. ${ }^{19}$ In two other studies, YAP expression was lower in prostate adenocarcinoma than in normal tissue. ${ }^{20,23}$ There was no significant tendency of YAP RNA expression profiles between prostate adenocarcinoma and normal tissue (Table 3).

Next, the relationship between YAP expression and the clinicopathologic factors were analyzed. For well-to-moderately differentiated adenocarcinoma (Gleason score of 6-7), 118 cases $(81.9 \%)$ were completely negative or weakly stained (intensity score of "low") for YAP (p<.01) (Table 4, Fig. 2E, F). In comparison, 23 cases of poorly differentiated adenocarcinoma $(52.3 \%)$ (Gleason score of 8-10) exhibited strong staining for YAP (intensity 
Table 2. Comparison of YAP expression between adenocarcinoma and normal prostate tissue adjacent to the adenocarcinoma

\begin{tabular}{lccc}
\hline Type & YAP negative/low expression & YAP high expression & p-value \\
\hline Normal prostate glands adjacent to adenocarcinoma & $163 / 188(86.7)$ & $25 / 188(13.3)$ & $>.05$ \\
Prostate adenocarcinoma & $139 / 188(73.9)$ & $49 / 188(26.1)$ & \\
\hline
\end{tabular}

Values are presented as number (\%).

YAP, Yes-associated protein.

Table 3. YAP mRNA expression in normal tissues and prostate adenocarcinoma from GEO profiles

\begin{tabular}{|c|c|c|c|c|c|}
\hline Study & Normal & No. of cases & Prostate cancer & No. of cases & $\mathrm{p}$-value \\
\hline Varambally et al..$^{18}$ & $3,797.54 \pm 2,444.70$ & 18 & $3,017.58 \pm 2,076.74$ & 21 & .29 \\
\hline Chandran et al..$^{19}$ & $44.48 \pm 21.65$ & 71 & $56.90 \pm 36.73$ & 91 & .01 \\
\hline Yu et al. ${ }^{20}$ & $1,280.71 \pm 445.43$ & 71 & $878.24 \pm 414.46$ & 91 & $<.01$ \\
\hline Satake et al. ${ }^{21}$ & $1,984.20 \pm 1,985.80$ & 3 & $1,254.13 \pm 1,500.77$ & 30 & .42 \\
\hline Planche et al..$^{22}$ & $8.43 \pm 1.54$ & 18 & $8.05 \pm 1.75$ & 18 & .11 \\
\hline Arredouani et al..23 & $1,139.21 \pm 806.37$ & 24 & $754.87 \pm 495.63$ & 39 & .02 \\
\hline Nanni et al..24 & $190.02 \pm 98.42$ & 8 & $189.80 \pm 64.38$ & 22 & .98 \\
\hline
\end{tabular}

Values are presented as mean \pm standard deviation

YAP, Yes-associated protein; GEO, Gene Expression Omnibus.

Table 4. Clinicopathologic features of the patients

\begin{tabular}{|c|c|c|c|c|}
\hline Characteristic & No. of patients & Low YAP expression & High YAP expression & $p$-value \\
\hline Age (yr) & & & & .74 \\
\hline$<70$ & 105 & 79 (75.2) & $26(24.8)$ & \\
\hline$\geq 70$ & 83 & $60(72.3)$ & $23(27.7)$ & \\
\hline Initial PSA (ng/mL) & & & & .44 \\
\hline$\leq 4.0$ & 7 & $5(71.4)$ & $2(28.6)$ & \\
\hline $4.1-10.0$ & 103 & $77(74.8)$ & $26(25.2)$ & \\
\hline $10.1-20.0$ & 44 & $29(65.9)$ & $15(34.1)$ & \\
\hline$>20$ & 34 & $28(82.4)$ & $6(17.6)$ & \\
\hline Tumor volume (g) & & & & .22 \\
\hline$<15$ & 122 & $94(77.0)$ & $28(23.0)$ & \\
\hline$\geq 15$ & 66 & 45 (68.2) & $21(31.8)$ & \\
\hline Tumor differentiation & & & & $<.01^{\mathrm{a}}$ \\
\hline Moderate to well differentiation (Gleason score 6-7) & 144 & $118(81.9)$ & $26(18.1)$ & \\
\hline Poor differentiation (Gleason score 8-10) & 44 & $21(47.7)$ & $23(52.3)$ & \\
\hline Pathologic stage & & & & .69 \\
\hline pT2 & 146 & $109(74.7)$ & $37(25.3)$ & \\
\hline pT3 & 42 & $30(71.4)$ & $12(28.6)$ & \\
\hline
\end{tabular}

Values are presented as number (\%).

YAP, Yes-associated protein; PSA, prostate-specific antigen.

aCochran Armitage trend test, $\mathrm{p}<.01$.

score of "high") (p<.01) (Table 4, Fig. 2G, H). However, no significant difference in YAP expression level was observed according to the age of the patient, tumor volume, preoperative serum PSA, or tumor stage (Table 4). A multivariate logistic regression model showed YAP expression (odds ratio [OR], 9.41; 95\% confidence interval [CI], 3.76 to 53.51), initial PSA (OR, 1.10; 95\% CI, 1.06 to 1.15 ), and pathologic stage (OR, 3.58; $95 \% \mathrm{CI}, 1.40$ to 9.42) to be significantly correlated with tumor differentiation (Table 5).

The BCR-free survival was significantly lower in patients with high YAP expressing cancers than those with negative/low YAP expressing cancers $(\mathrm{p}=.02)$ (Fig. 3). In addition, univariate analysis showed that the Gleason grade (hazard ratio, 5.02; $\mathrm{p}<.01$ ) and high YAP expression (hazard ratio, 1.919; $\mathrm{p}=.02$ ) were associated with BCR. Furthermore, multivariate analysis using a Cox regression model demonstrated that the Gleason grade $(\mathrm{p}<.01)$ and $\mathrm{T}$ stage were independent prognostic factors for BCR. However YAP expression was not a significantly independent prognostic factor for BCR in the Cox proportional hazards model (Table 6). 


\section{DISCUSSION}

YAP regulates cell proliferation, stem behavior and regeneration. ${ }^{15}$ Promotion of cell stemness and proliferation are important factors for cancer development and regeneration. ${ }^{15}$ YAP can be

Table 5. Significant risk factors for tumor differentiation in patients with prostate adenocarcinoma determined by multivariate logistic regression model

\begin{tabular}{lcc}
\hline \multirow{2}{*}{ Factor } & \multicolumn{2}{c}{ Multivariate } \\
\cline { 2 - 3 } & Odds ratio $(95 \% \mathrm{Cl})$ & $\mathrm{p}$-value \\
\hline Age & $1.24(0.54-2.89)$ & .61 \\
Initial PSA & $1.10(1.06-1.15)$ & $<.01$ \\
Tumor volume & $1.08(0.44-2.64)$ & .87 \\
YAP expression & $9.41(3.76-23.51)$ & $<.01$ \\
Pathologic stage & $3.58(1.40-9.42)$ & $<.01$ \\
\hline
\end{tabular}

$\mathrm{Cl}$, confidence interval; PSA, prostate specific antigen; YAP, Yes-associated protein.

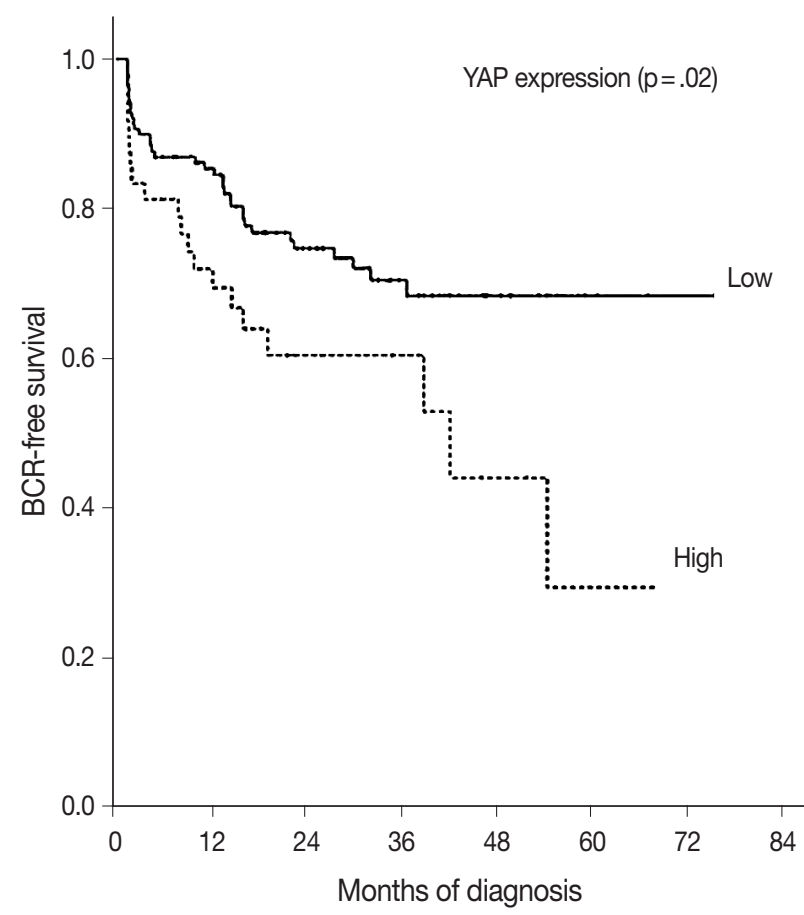

Fig. 3. Kaplan-Meier estimates of biochemical recurrence. Graph of the curve for progression-free survival in prostate adenocarcinoma patients, according to Yes-associated protein (YAP) expression $(p=.02)$. used during the development of cancer and other phenotypes during reprogramming of mature and differentiated cells. ${ }^{15}$

As noted above, the expression of YAP via immunohistochemical staining showed no statistically significant difference between prostate adenocarcinoma and adjacent normal tissues. Similarly, in GEO data profiles, YAP mRNA was not statistically higher in prostate adenocarcinoma than in normal tissue. This suggested that YAP expression is not associated with tumorigenesis of prostate adenocarcinoma. YAP and/or TAZ knockdown in human colorectal cancer cell lines suppresses their growth and ability to trigger tumor formation after injection in mice. ${ }^{25}$ On the other hand, YAP has been reported to be functionally implicated to tumorigenesis in other human cancers. Liver-specific YAP overexpression in transgenic mice leads to hepatomegaly and development of human hepatocellular carcinoma. ${ }^{26}$ Similar results were obtained by delivering a phosphomutant form of YAP in the mouse liver using transposon-mediated hydrodynamic transfection. ${ }^{27}$ YAP or TAZ knockdown strongly reduces subcutaneous tumor growth of human and mouse hepatocellular carcinoma cell lines. ${ }^{28}$ Functionally, pancreasspecific YAP knockout abrogates tumor progression from early pancreatic intraepithelial neoplasia lesions to pancreatic ductal adenocarcinoma in a mouse model of pancreatic cancer. ${ }^{29}$

Poorly differentiated prostate adenocarcinoma cells stained more strongly for YAP than in moderately to well-to-moderately differentiated prostate adenocarcinoma. YAP immunoreactivity was inversely associated with the differentiation of prostate adenocarcinoma. In addition, YAP expression was found to be a factor affecting tumor differentiation. This finding indicates that there is a meaningful inverse relationship between YAP expression and differentiation of prostate adenocarcinoma, which suggests that YAP may play a role in the differentiation of prostate adenocarcinoma.

Hu et al. ${ }^{17}$ showed that down-regulation of YAP expression in prostate adenocarcinoma correlates with an increase in Gleason score, and this study showed differences from the results. It can be explained for several factors. First, the expression of YAP showed intratumoral heterogeneity in prostate adenocarcinoma. In tumors with heterogeneous expression, many tissue cores are

Table 6. Univariate and multivariate analyses for predictive factors in patients with prostate adenocarcinoma

\begin{tabular}{|c|c|c|c|c|}
\hline \multirow{2}{*}{ Factor } & \multicolumn{2}{|c|}{ Univariate } & \multicolumn{2}{|c|}{ Multivariate } \\
\hline & Hazard ratio $(95 \% \mathrm{Cl})$ & $p$-value & Hazard ratio $(95 \% \mathrm{Cl})$ & $p$-value \\
\hline Gleason grade group & $5.02(2.97-8.50)$ & $<.01$ & $4.33(0.56-1.87)$ & $<.01$ \\
\hline High YAP expression & $1.92(1.11-3.32)$ & .02 & $1.03(0.99-3.08)$ & .93 \\
\hline
\end{tabular}

$\mathrm{Cl}$, confidence interval; YAP, Yes-associated protein. 
required to get comprehensive information. Hu et al. ${ }^{17}$ used tissue microarray (TMA) slides with two cores of intratumoral and peritumoral tissue per slide. In this study, we used we used a representative slide instead of TMA slide. Second, the interpretation method of YAP expression was different. It was estimated according to the distribution and intensity of positive staining in this study according to Steinhardt et al. ${ }^{8}$ However $\mathrm{Hu} e t$ al. ${ }^{17}$ interpreted it as three categories, only cytoplasmic, only nuclear, both nuclear and cytoplasmic. Those factors might affect the difference of the results of this study and $\mathrm{Hu}$ et al..$^{17}$

Enhanced YAP activity tends to promote stem cells and progenitor cells, while inhibiting differentiation. ${ }^{30}$ Immunohistochemical studies on human hepatocellular carcinoma samples showed that elevated expression of YAP or TAZ correlates with poor differentiation of cancer cells and poor outcomes. ${ }^{31}$ In datasets of patients with breast cancer, an elevated expression of gene signatures for YAP/TAZ activity correlate with high histological grades, enrichment of stem cell signatures, tendency to metastasize, and poor outcomes. ${ }^{10,32}$ Prostate stem cell antigen expression identified through immunohistochemical staining and in situ hybridization is significantly increased in human PCs with a high Gleason score. ${ }^{33}$ Considering that YAP is generally associated with cancer stem cells, we can further compare the expression of YAP and prostate stem cell antigens with differentiation stages of prostate adenocarcinoma. Once the relationship between PC stem cells and YAP and its mechanisms of action are revealed, it could be further explored as a new therapeutic target.

In this study, YAP immunoreactivity was not associated with the $\mathrm{BCR}$ of prostate adenocarcinoma. The univariate analysis was statistically significant, but not the multivariate analysis. This difference between the univariate and multivariate analyses might be attributed to the contribution of the Gleason score. Therefore, YAP immunoreactivity for prostate adenocarcinoma was not useful for predicting adverse outcomes. Expression of YAP in a variety of human cancers has been reported to be associated with a poor prognosis. High levels of YAP expression were found to be associated with poor outcomes in four datasets of colorectal cancer patients, and were positively correlated with cetuximab resistance. ${ }^{34}$ YAP mRNA and protein levels have been shown to be upregulated in gastric adenocarcinoma, and YAP protein expression and nuclear localization were associated with poor patient outcomes. ${ }^{35}$

There are several limitations to this study. First, this study investigated the relationship between YAP expression and clinicopathologic factors in prostate adenocarcinoma based on the morphological expression of the cancer. No functional studies of YAP have been performed in relation to tumorigenesis of prostate adenocarcinoma. In addition, this study did not show a direct functional relationship between YAP expression and the differentiation pathway of prostate adenocarcinoma. The second limitation was that the tumor differentiation was interpreted in a two-tier system: well to moderate differentiation (Gleason score of 6-7) or poorly-differentiation (Gleason score of 8-10). The reason for this was that the number of patients subclassified by their Gleason score was largely uneven. The number of patients with Gleason score of 6 was 39 , of 7 was 105 , of 8 was 26, of 9 was 17 , and of 10 was 1. If a larger cohort of patients was included in this study, the study might have had more specific results.

This study demonstrates that YAP is highly expressed in poorly differentiated prostate adenocarcinoma compared to welldifferentiated or moderately differentiated prostate adenocarcinoma. However, YAP expression was not associated with BCR in the Cox proportional hazards model.

\section{Conflicts of Interest}

No potential conflict of interest relevant to this article was reported.

\section{REFERENCES}

1. Diallo JS, Aldejmah A, Mouhim AF, et al. Co-assessment of cytoplasmic and nuclear androgen receptor location in prostate specimens: potential implications for prostate cancer development and prognosis. BJU Int 2008; 101: 1302-9.

2. Joung JY, Lim J, Oh CM, et al. Current trends in the incidence and survival rate of urological cancers in Korea. Cancer Res Treat 2016 Sep 23 [Epub]. https://doi.org/10.4143/crt.2016.139.

3. Zhang L, Yang S, Chen X, et al. The hippo pathway effector YAP regulates motility, invasion, and castration-resistant growth of prostate cancer cells. Mol Cell Biol 2015; 35: 1350-62.

4. Epstein JI, Allsbrook WC Jr, Amin MB, Egevad LL; ISUP Grading Committee. The 2005 International Society of Urological Pathology (ISUP) Consensus Conference on Gleason Grading of Prostatic Carcinoma. Am J Surg Pathol 2005; 29: 1228-42.

5. McMenamin ME, Soung P, Perera S, Kaplan I, Loda M, Sellers WR. Loss of PTEN expression in paraffin-embedded primary prostate cancer correlates with high Gleason score and advanced stage. Cancer Res 1999; 59: 4291-6.

6. Edgar BA. From cell structure to transcription: Hippo forges a new path. Cell 2006; 124: 267-73.

7. Lai ZC, Wei X, Shimizu T, et al. Control of cell proliferation and 
apoptosis by mob as tumor suppressor, mats. Cell 2005; 120: 675-85.

8. Steinhardt AA, Gayyed MF, Klein AP, et al. Expression of Yes-associated protein in common solid tumors. Hum Pathol 2008; 39: 1582-9.

9. Wang Y, Dong Q, Zhang Q, Li Z, Wang E, Qiu X. Overexpression of yes-associated protein contributes to progression and poor prognosis of non-small-cell lung cancer. Cancer Sci 2010; 101: 1279-85.

10. Cordenonsi M, Zanconato F, Azzolin L, et al. The Hippo transducer TAZ confers cancer stem cell-related traits on breast cancer cells. Cell 2011; $147: 759-72$

11. Di Agostino S, Sorrentino G, Ingallina E, et al. YAP enhances the proproliferative transcriptional activity of mutant $\mathrm{p} 53$ proteins. EMBO Rep 2016; 17: 188-201.

12. Lee KW, Lee SS, Kim SB, et al. Significant association of oncogene YAP1 with poor prognosis and cetuximab resistance in colorectal cancer patients. Clin Cancer Res 2015; 21: 357-64.

13. Kim GJ, Kim H, Park YN. Increased expression of Yes-associated protein 1 in hepatocellular carcinoma with stemness and combined hepatocellular-cholangiocarcinoma. PLoS One 2013; 8: e75449.

14. Morvaridi S, Dhall D, Greene MI, Pandol SJ, Wang Q. Role of YAP and TAZ in pancreatic ductal adenocarcinoma and in stellate cells associated with cancer and chronic pancreatitis. Sci Rep 2015; 5: 16759.

15. Johnson R, Halder G. The two faces of Hippo: targeting the Hippo pathway for regenerative medicine and cancer treatment. Nat Rev Drug Discov 2014; 13: 63-79.

16. Grasso CS, Wu YM, Robinson DR, et al. The mutational landscape of lethal castration-resistant prostate cancer. Nature 2012; 487: 239-43.

17. Hu X, Jia Y, Yu J, Chen J, Fu Q. Loss of YAP protein in prostate cancer is associated with Gleason score increase. Tumori 2015; 101: 189-93.

18. Varambally S, Yu J, Laxman B, et al. Integrative genomic and proteomic analysis of prostate cancer reveals signatures of metastatic progression. Cancer Cell 2005; 8: 393-406.

19. Chandran UR, Ma C, Dhir R, et al. Gene expression profiles of prostate cancer reveal involvement of multiple molecular pathways in the metastatic process. BMC Cancer 2007; 7: 64.

20. Yu YP, Landsittel D, Jing L, et al. Gene expression alterations in prostate cancer predicting tumor aggression and preceding development of malignancy. J Clin Oncol 2004; 22: 2790-9.

21. Satake H, Tamura K, Furihata M, et al. The ubiquitin-like molecule interferon-stimulated gene 15 is overexpressed in human prostate cancer. Oncol Rep 2010; 23: 11-6.

22. Planche A, Bacac M, Provero P, et al. Identification of prognostic molecular features in the reactive stroma of human breast and pros- tate cancer. PLoS One 2011; 6: e18640.

23. Arredouani MS, Lu B, Bhasin M, et al. Identification of the transcription factor single-minded homologue 2 as a potential biomarker and immunotherapy target in prostate cancer. Clin Cancer Res 2009; 15: 5794-802.

24. Nanni S, Priolo C, Grasselli A, et al. Epithelial-restricted gene profile of primary cultures from human prostate tumors: a molecular approach to predict clinical behavior of prostate cancer. Mol Cancer Res 2006; 4: 79-92.

25. Rosenbluh J, Nijhawan D, Cox AG, et al. beta-Catenin-driven cancers require a YAP1 transcriptional complex for survival and tumorigenesis. Cell 2012; 151: 1457-73.

26. Dong J, Feldmann G, Huang J, et al. Elucidation of a universal sizecontrol mechanism in Drosophila and mammals. Cell 2007; 130: 1120-33.

27. Shen S, Guo X, Yan H, et al. A miR-130a-YAP positive feedback loop promotes organ size and tumorigenesis. Cell Res 2015; 25: 997-1012.

28. Xiao W, Wang J, Ou C, et al. Mutual interaction between YAP and c-Myc is critical for carcinogenesis in liver cancer. Biochem Biophys Res Commun 2013; 439: 167-72.

29. Zhang W, Nandakumar N, Shi Y, et al. Downstream of mutant KRAS, the transcription regulator YAP is essential for neoplastic progression to pancreatic ductal adenocarcinoma. Sci Signal 2014; 7: ra42.

30. Zanconato F, Cordenonsi M, Piccolo S. YAP/TAZ at the roots of cancer. Cancer Cell 2016; 29: 783-803.

31. Guo Y, Pan Q, Zhang J, et al. Functional and clinical evidence that TAZ is a candidate oncogene in hepatocellular carcinoma. J Cell Biochem 2015; 116: 2465-75.

32. Bartucci M, Dattilo R, Moriconi C, et al. TAZ is required for metastatic activity and chemoresistance of breast cancer stem cells. Oncogene 2015; 34: 681-90.

33. Han KR, Seligson DB, Liu X, et al. Prostate stem cell antigen expression is associated with gleason score, seminal vesicle invasion and capsular invasion in prostate cancer. J Urol 2004; 171: 1117-21.

34. Kim DH, Kim SH, Lee OJ, et al. Differential expression of Yes-associated protein and phosphorylated Yes-associated protein is correlated with expression of Ki-67 and phospho-ERK in colorectal adenocarcinoma. Histol Histopathol 2013; 28: 1483-90.

35. Hu X, Xin Y, Xiao Y, Zhao J. Overexpression of YAP1 is correlated with progression, metastasis and poor prognosis in patients with gastric carcinoma. Pathol Oncol Res 2014; 20: 805-11. 